\title{
QUANTUM MECHANICAL SPECTRAL ENGINEERING BY SCALING INTERTWINING
}

\author{
DAVID J. FERNÁNDEZ C..$^{\dagger}$ and HARET C. ROSU $\ddagger$ \\ † Departamento de Fúsica, CINVESTAV-IPN, Apdo Postal 14-740, 07000 México Distrito Federal, Mexico \\ ¥ Instituto de Física de la Universidad de Guanajuato, Apdo Postal E-143, León, Guanajuato, Mexico
}

\begin{abstract}
Using the concept of spectral engineering we explore the possibilities of building potentials with prescribed spectra offered by a modified intertwining technique involving operators which are the product of a standard first-order intertwiner and a unitary scaling. In the same context we study the iterations of such transformations finding that the scaling intertwining provides a different and richer mechanism in designing quantum spectra with respect to that given by the standard intertwining.
\end{abstract}

PACS number(s): 03.65.Fd, 03.65.Ge, 11.30.Pb

Physica Scripta 64 (2001) 177-183

\section{INTRODUCTION}

The remarkable success in producing artificial systems with controllable physical properties (quantum wells and dots in materials science [1], traps in atomic physics [2] [3], spatially confined quantum systems [4] among others) has stimulated the research of the so called quantum engineering (see e.g., the recent review [5]). For instance, the control and manipulation of effects such as diffraction, widening, interference, etc., of either specific or arbitrary quantum states have been longly addressed from both theoretical and experimental viewpoints [6] - [8]. In the same spirit, the "technological" idea of designing potentials with prescribed quantum spectra [9], a subject that one may call spectral engineering is worth of investigating. Some progress in this area can be done by restricting the construction to potentials whose spectra are slightly different from a given initial one through the usage of the well-known algebraic procedures provided by the factorization method [10] - 12], supersymmetric quantum mechanics [13] - 115] and other related techniques [16] [22]. It is worth noticing that these developments can be recovered from the equivalent intertwining technique [23], which provides a better understanding of the generating process [18 - 20]. However, nowadays it becomes apparent the need of novel theoretical techniques additional to the standard intertwining in order to manufacture quantum spectra and get further insight for new technological, device-oriented possibilities at nanoscopic and mesoscopic scales.

With this motivation, we present here a modified intertwining as an additional mechanism to generate potentials with prescribed spectra. The modification will consist in replacing the standard intertwiner by an operator which is the product of a standard intertwiner and a scale transformation. This constitutes a variant of the method used to analize the so-called self-similar potentials [24,25] (for a review see [26]). Mathematically speaking we will see that this simple change is essentially equivalent to the standard intertwining. However, we will find that the spectra of the potentials generated by the scaling intertwining can be manipulated (when the scaling parameters and the position of the new level are changed) in an essentially different way from the manipulation possibilities provided by the standard intertwining.

The paper is organized as follows. The standard first order intertwining will be introduced in section 2, while the higher order case as an iterative procedure of the first order one will be presented in section 3 . In both sections the possibilities for the spectral engineering offered by each technique will be discussed. The scaling intertwining as a generalization of the first order method of section 2 will be the subject of section 4 and its iterations will be addressed in section 5. At section 6 the spectral possibilities offered by all these techniques will be illustrated by means of the harmonic oscillator. Finally, section 7 contains some concluding remarks.

\section{FIRST ORDER INTERTWINING TECHNIQUE}

In the standard intertwining one looks for an operator $A_{1}^{+}$connecting two Hamiltonians as

$$
H_{1} A_{1}^{+}=A_{1}^{+} H_{0},
$$

where

$$
H_{i}=-\frac{1}{2} \frac{d^{2}}{d x^{2}}+V_{i}(x), \quad i=0,1 .
$$

The first order intertwining uses for $A_{1}^{+}$the operator

$$
A_{1}^{+}=\frac{1}{\sqrt{2}}\left(-\frac{d}{d x}+\alpha_{1}(x, \epsilon)\right),
$$

leading to the standard Riccati equation for $\alpha_{1}(x, \epsilon)$ associated to the given initial potential 


$$
\alpha_{1}^{\prime}(x, \epsilon)+\alpha_{1}^{2}(x, \epsilon)=2\left(V_{0}(x)-\epsilon\right),
$$

where the prime denotes the derivative with respect to $x$. The new potential $V_{1}(x)$ is determined according to:

$$
V_{1}(x)=V_{0}(x)-\alpha_{1}^{\prime}(x, \epsilon),
$$

whenever one is able to find a solution to (4) for given $V_{0}(x)$ and $\epsilon$. The factorization energy $\epsilon \in \mathbf{R}$ is quite important for the properties of $V_{1}(x)$, as it is clear after substituting $\alpha_{1}(x, \epsilon)=[\ln \psi(x)]^{\prime}$ in (4) leading to

$$
H_{0} \psi(x)=-\frac{1}{2} \psi^{\prime \prime}(x)+V_{0}(x) \psi(x)=\epsilon \psi(x) .
$$

Although similar to the standard eigenvalue equation for $H_{0}$, notice that $\psi(x)$ in (6) is not necessarily normalizable, but it should not have zeros in order to avoid supplementary singularities of $V_{1}(x)$ with respect to those of $V_{0}(x)$. As is well known, for $\epsilon>E_{0}$ ( $E_{0}$ is the ground state energy of $\left.H_{0}\right) \psi(x)$ will always have zeros. However, if $\epsilon \leq E_{0}$ it is possible to make $\psi(x)$ to have no zeros by adjusting the ratio of the two constants in the general solution of (6), resulting in a physically meaningful $V_{1}(x)$ as explained for example by Sukumar 13. The spectrum of $V_{1}(x)$ consists of the sequence of levels $E_{n}$ of the initial Hamiltonian plus a new 'ground state' at $\epsilon$. The scheme becomes complete after realizing that $H_{0}$ and $H_{1}$ are factorized in terms of $A_{1}$ and $A_{1}^{+}$as follows

$$
H_{0}=A_{1} A_{1}^{+}+\epsilon, \quad H_{1}=A_{1}^{+} A_{1}+\epsilon .
$$

Let us notice that equation (5) is equivalent to $H_{1}=$ $H_{0}-\alpha_{1}^{\prime}(x, \epsilon)$. Thus, the intertwining relationship (1) can be put in the commutator form $\left[H_{0}, A_{1}^{+}\right]=\alpha_{1}^{\prime}(x, \epsilon) A_{1}^{+}$.

The possibilities for designing potentials with prescribed spectra offered by the standard first order intertwining are restricted by our skills to solve the Riccati equation (4) or its equivalent (6) for different values of the factorization energy $\epsilon$. A simple situation arises if we are able to solve (4) for $\epsilon$ sweeping an interval $I=\left(\epsilon_{\mathrm{L}}, \epsilon_{\mathrm{R}}\right)$. By varying $\epsilon$ in such a domain we are 'manipulating' the spectra of the corresponding potentials $V_{1}(x)$ in such a way that the excited levels $E_{n}$ remain fixed but the ground state level $\epsilon$ is moving with respect to those levels. This is a modest but interesting contribution to the spectral engineering offered by the first order intertwining.

\section{HIGHER ORDER INTERTWINING TECHNIQUE}

In order to improve the spectral controllability, it would be important to explore additional techniques to find solvable potentials with known spectra. A useful one consists of iterations of the first order intertwining technique of section 2 [16]- 20]. Suppose that, departing from a solvable Hamiltonian $H_{0}$, we have constructed a chain of Hamiltonians of form $(2),\left\{H_{i}, i=1, \ldots, k\right\}$, that are pair-intertwined according to

$$
H_{i} A_{i}^{+}=A_{i}^{+} H_{i-1}, \quad i=1, \ldots, k,
$$

where

$$
A_{i}^{+}=\frac{1}{\sqrt{2}}\left(-\frac{d}{d x}+\alpha_{i}\left(x, \epsilon_{i}\right)\right) .
$$

Thus, $\alpha_{i}\left(x, \epsilon_{i}\right)$ must satisfy a Riccati equation similar to (4),

$$
\alpha_{i}^{\prime}\left(x, \epsilon_{i}\right)+\alpha_{i}^{2}\left(x, \epsilon_{i}\right)=2\left[V_{i-1}(x)-\epsilon_{i}\right],
$$

and $V_{i}(x)$ is related to $V_{i-1}(x)$ according to

$$
V_{i}(x)=V_{i-1}(x)-\alpha_{i}^{\prime}\left(x, \epsilon_{i}\right) .
$$

It is simple to show that $\alpha_{i}\left(x, \epsilon_{i}\right)$ can be obtained from $\alpha_{i-1}$ evaluated at two different factorization energies $\epsilon_{i}, \epsilon_{i-1}$ through the following finite difference formula [18]- [20]:

$\alpha_{i}\left(x, \epsilon_{i}\right)=-\alpha_{i-1}\left(x, \epsilon_{i-1}\right)-\frac{2\left(\epsilon_{i-1}-\epsilon_{i}\right)}{\alpha_{i-1}\left(x, \epsilon_{i-1}\right)-\alpha_{i-1}\left(x, \epsilon_{i}\right)}$.

Let us notice that this equation was previously obtained by Adler when studying Bäcklund transformations for Painlevé equations [27]. By iterating this formula in the subindex $i$ it is clear that the final potential $V_{k}(x)=V_{k-1}(x)-\alpha_{k}^{\prime}\left(x, \epsilon_{k}\right)$ is determined by $k$ solutions $\alpha_{1}\left(x, \epsilon_{i}\right), i=1, \ldots, k$ to the initial Riccati equation (4) associated to the $k$ factorization energies $\epsilon_{1}, \ldots, \epsilon_{k}$. The spectrum of $V_{k}(x)$ consists of the levels $E_{n}$ of the original Hamiltonian $H_{0}$ plus $k$ new levels below the ground state energy level $E_{0}$ of $H_{0}$ (by simplicity ordered according to $\left.\epsilon_{k}<\epsilon_{k-1}<\cdots<\epsilon_{1}<E_{0}\right)$.

As in the first order intertwining, in the higher order case the spectral engineering depends on how many solutions we are able to find for the initial Riccati equation (4) as well as on the positions of the $k$ different factorization energies $\epsilon_{1}, \ldots, \epsilon_{k}$. If we are able to find solutions only for isolated values of $\epsilon_{1}, \ldots, \epsilon_{k}$, we can hardly manipulate the spectrum of the potentials $V_{k}(x)$. The really interesting situation arises if we are able to find solutions for $\epsilon$ sweeping an interval $I$. Let us choose $k$ of these solutions $\alpha_{1}\left(x, \epsilon_{i}\right), i=1, \ldots, k$. By moving those $k$ values in such a way that always $\epsilon_{i} \in I$ and the previous ordering of the $\epsilon_{i}$ 's is respected, we will be manipulating the spectrum of $V_{k}(x)$ so that the levels $E_{n}$ are fixed but the $k$ ones $\epsilon_{i}, i=1, \ldots, k$ will be moving in a way that could be selected according to some specific purposes. This provides more freedom for controlling quantum spectra than that offered by the first order intertwining. Moreover, we 
shall see below that it is possible to design different tools based on the same mathematical formalism of section 2 which will provide new mechanisms to manipulate quantum spectra.

\section{THE SCALING INTERTWINING METHOD}

Let us consider a slight generalization of the first-order intertwining of section 2 by maintaining Eq. (1) but introducing now a 'deforming' parameter $q_{1}$ into $H_{1}$ as $H_{1}=$ $q_{1}^{2} H_{0}-f(x)$, and substituting the intertwining operator (3) by the same standard intertwiner times a scaling unitary operator $U_{1} \equiv e^{i \frac{\lambda_{1}}{2}(\hat{x} \hat{p}+\hat{p} \hat{x})}=e^{\frac{\lambda_{1}}{2}\left(x \frac{d}{d x}+\frac{d}{d x} x\right)}, \lambda_{1} \in \mathbf{R}$ (see, e.g., [24- 28):

$$
A_{\lambda_{1}}^{+}=\frac{1}{\sqrt{2}}\left(-\frac{d}{d x}+\alpha_{1}\left(x, \epsilon_{1}\right)\right) U_{1} .
$$

Multiplying Eq. (1) by $U_{1}^{+}$to the right one gets

$$
\begin{aligned}
& {\left[q_{1}^{2} H_{0}-f(x)\right]\left[-\frac{d}{d x}+\alpha_{1}\left(x, \epsilon_{1}\right)\right]} \\
& =\left[-\frac{d}{d x}+\alpha_{1}\left(x, \epsilon_{1}\right)\right] U_{1} H_{0} U_{1}^{+},
\end{aligned}
$$

and making equal on both sides of (14) the coefficients of the various corresponding powers of the derivative operator $\frac{d}{d x}$ leads to

$$
\begin{gathered}
q_{1}^{2}=e^{-2 \lambda_{1}} \\
q_{1}^{2} \alpha_{1}^{\prime}\left(x, \epsilon_{1}\right)+q_{1}^{2} V_{0}(x)-f(x)=V_{0}\left(e^{\lambda_{1}} x\right) \\
-\frac{q_{1}^{2}}{2} \alpha_{1}^{\prime \prime}\left(x, \epsilon_{1}\right)+\left[q_{1}^{2} V_{0}(x)-f(x)\right] \alpha_{1}\left(x, \epsilon_{1}\right) \\
=\alpha_{1}\left(x, \epsilon_{1}\right) V_{0}\left(e^{\lambda_{1}} x\right)-V_{0}^{\prime}\left(e^{\lambda_{1}} x\right) .
\end{gathered}
$$

After substituting $\lambda_{1}=-\ln q_{1}$, Eq. (16) reads

$$
f(x)=q_{1}^{2} \alpha_{1}^{\prime}\left(x, \epsilon_{1}\right)+q_{1}^{2} V_{0}(x)-V_{0}\left(x / q_{1}\right) .
$$

Notice the non-local character of equation (18) for $q_{1} \neq 1$ in contrast with the local nature of the standard intertwining $\left(q_{1}=1\right)$ for which $f(x)=\alpha_{1}^{\prime}\left(x, \epsilon_{1}\right)$ : in the scaling intertwining the original potential at two different points, $x$ and $x / q_{1}$, interfere with $\alpha_{1}^{\prime}\left(x, \epsilon_{1}\right)$ in order to provide $f(x)$. This difference arises even when $V_{0}(x)$ is a homogeneous function of degree $d$ for which (18) reduces to the more local form $f(x)=q_{1}^{2} \alpha_{1}^{\prime}\left(x, \epsilon_{1}\right)+\left(q_{1}^{2}-\right.$ $\left.q_{1}^{-d}\right) V_{0}(x)$. When $d=-2$ one gets $f(x)=q_{1}^{2} \alpha_{1}^{\prime}\left(x, \epsilon_{1}\right)$ which is the closest one can reach to the standard intertwining. Substituting $\lambda_{1}=-\ln q_{1}$ and (18) in Eq. (17) and integrating it we get the Riccati equation

$$
q_{1}^{2} \alpha_{1}^{\prime}\left(x, \epsilon_{1}\right)+q_{1}^{2} \alpha_{1}^{2}\left(x, \epsilon_{1}\right)=2\left[V_{0}\left(x / q_{1}\right)-\epsilon_{1}\right]
$$

where, as it will be clear below, it is convenient to choose the integration constant equal to one of the factorization energies $\epsilon_{1}$ of (4). Now, if one makes the change of variable $y=x / q_{1}$ and denotes $\tilde{\alpha}_{1}\left(y, \epsilon_{1}\right) \equiv q_{1} \alpha_{1}\left(q_{1} y, \epsilon_{1}\right)$ one easily gets

$$
\frac{d \tilde{\alpha}_{1}\left(y, \epsilon_{1}\right)}{d y}+\tilde{\alpha}_{1}^{2}\left(y, \epsilon_{1}\right)=2\left(V_{0}(y)-\epsilon_{1}\right) .
$$

This is again the Riccati equation (4) with factorization energy $\epsilon_{1}$. Therefore, the same Riccati solution used in the standard intertwining (Eqs. (1) to (7)) can be used in the scaling intertwining of Eqs. $(1,13-20)$ as well in order to generate solvable potentials with known spectra. The eigenfunctions of $\tilde{H}_{1} \equiv q_{1}^{-2} H_{1}=H_{0}-q_{1}^{-2} f(x)$ are proportional to the action of $A_{\lambda_{1}}^{+}$on the eigenfunctions of $H_{0}$ due to the fact that the two Schrödinger Hamiltonians $H_{0}$ and $\tilde{H}_{1}$ are intertwined in the slightly generalized way

$$
\tilde{H}_{1} A_{\lambda_{1}}^{+}=q_{1}^{-2} A_{\lambda_{1}}^{+} H_{0}
$$

The potential associated to $\tilde{H}_{1}$ takes the form

$$
\tilde{V}_{1}(x)=V_{0}(x)-q_{1}^{-2} f(x)=q_{1}^{-2} V_{0}\left(x / q_{1}\right)-\alpha_{1}^{\prime}\left(x, \epsilon_{1}\right) .
$$

The corresponding eigenvalues are $\left\{q_{1}^{-2} \epsilon_{1}, q_{1}^{-2} E_{n}\right\}$, where $q_{1}^{-2} \epsilon_{1}$ is the ground state energy of $\tilde{H}_{1}$ associated to the eigenfunction $\tilde{\psi}_{\epsilon_{1}}^{(1)}(x) \propto \exp \left(-\int_{0}^{x} \alpha_{1}\left(\xi, \epsilon_{1}\right) d \xi\right)$. The factorizations of $H_{0}$ and $\tilde{H}_{1}$ in terms of $A_{\lambda_{1}}$ and $A_{\lambda_{1}}^{+}$become

$$
H_{0}=q_{1}^{2} A_{\lambda_{1}} A_{\lambda_{1}}^{+}+\epsilon_{1}, \quad \tilde{H}_{1}=A_{\lambda_{1}}^{+} A_{\lambda_{1}}+q_{1}^{-2} \epsilon_{1} .
$$

The spectral engineering skills depend once again on the kind of solutions to the Riccati equations (4) and (20) we are able to get. If we find a solution for an isolated value of the factorization energy $\epsilon_{1}$, the potentials $\tilde{V}_{1}(x)$ will have the spectrum $\left\{q_{1}^{-2} \epsilon_{1}, q_{1}^{-2} E_{n}\right\}$, and by varying $q_{1}$ we will be just scaling the basic spectrum $\left\{\epsilon_{1}, E_{n}\right\}$ at $q_{1}=1$ generated by means of the standard intertwining when a new level is created at $\epsilon_{1}$ starting from $V_{0}(x)$. However, notice that solutions of (20) for $\epsilon_{1}$ sweeping a real interval $I=\left(\epsilon_{\mathrm{L}}, \epsilon_{\mathrm{R}}\right)$, where $\epsilon_{\mathrm{L}}$ and $\epsilon_{\mathrm{R}}$ have the same sign, suggest choosing $\epsilon_{1}=q_{1}^{2} \epsilon_{1 \mathrm{~F}}$ with $\epsilon_{1 \mathrm{~F}} \in I$ fixed, and varying $q_{1}$ in such a way as to keep $\epsilon_{1} \in I$. Thus, we will manipulate the spectrum of $\tilde{V}_{1}(x),\left\{\epsilon_{1 \mathrm{~F}}, q_{1}^{-2} E_{n}\right\}$ by scaling by a factor $q_{1}^{-2}$ the excited levels $E_{n}$ while the ground state energy remains fixed. This effect is opposite to that produced by the standard intertwining where the excited levels are static while the changing one is the ground state level. Thus, we have at hand two different tools increasing our skills in the spectral engineering 
that could be useful in various physical situations. Moreover, as we shall see below the scaling intertwining can be iterated in a similar way as the standard intertwining.

In order to implement the iterations of this technique, let us notice that the Hamiltonian $H_{1}=q_{1}^{2} H_{0}-f(x)$ expressed in the coordinate $y=x / q_{1}$ acquires the standard Schrödinger form

$$
H_{1}=-\frac{1}{2} \frac{d^{2}}{d y^{2}}+V_{1}(y)
$$

where

$$
V_{1}(y) \equiv q_{1}^{2} \tilde{V}_{1}\left(q_{1} y\right)=V_{0}(y)-\frac{d \tilde{\alpha}_{1}\left(y, \epsilon_{1}\right)}{d y} .
$$

These formulae clarify better the scaling intertwining mechanism: the modified intertwiner $A_{\lambda_{1}}^{+}$connects two almost isospectral Hamiltonians $H_{0}$ and $H_{1}$, creating a new level for $H_{1}$ at $\epsilon_{1}$ and simultaneously changing the coordinate $x$ to $y=x / q_{1}$. When we come back to the original coordinate $x$ and scale the energy, we arrive at the Hamiltonian $\tilde{H}_{1}$ having the standard Schrödinger form in terms of $x$. In this way we get the modified intertwining of Eq. (21) which leads to the spectral properties previously discussed.

\section{ITERATIVE SCALING INTERTWINING}

Let us restrict our considerations to two iterations of the scaling intertwining. The generalization to higher iterative orders follows straightforwardly from the pair of transformations we shall study in this section. During the first step we will have $H_{1} A_{\lambda_{1}}^{+}=A_{\lambda_{1}}^{+} H_{0}$, as in the previous section, while in the second step we will have $H_{2} A_{\lambda_{2}}^{+}=A_{\lambda_{2}}^{+} H_{1}$. Taking into account the remark at the end of section 4, it is clear that in the second step the Hamiltonian $H_{1}$ of Eq. (24) 'is changed' into a new one $H_{2}$ expressed in a different coordinate $z=y / q_{2}$ as follows

$$
H_{2}=-\frac{1}{2} \frac{d^{2}}{d z^{2}}+V_{2}(z) .
$$

The corresponding scaling intertwining operator is now:

$$
A_{\lambda_{2}}^{+}=\frac{1}{\sqrt{2}}\left(-\frac{d}{d y}+\alpha_{2}\left(y, \epsilon_{2}\right)\right) U_{2},
$$

where $U_{2} \equiv e^{i \frac{\lambda_{2}}{2}(\hat{x} \hat{p}+\hat{p} \hat{x})}=e^{\frac{\lambda_{2}}{2}\left(y \frac{d}{d y}+\frac{d}{d y} y\right)}$. The appropriate reading of equations (14-18) leads to $\lambda_{2}=-\ln q_{2}$ and an equation analogue to (19)

$$
q_{2}^{2} \frac{d \alpha_{2}\left(y, \epsilon_{2}\right)}{d y}+q_{2}^{2} \alpha_{2}^{2}\left(y, \epsilon_{2}\right)=2\left[V_{1}\left(y / q_{2}\right)-\epsilon_{2}\right] .
$$

With the change of variables $z=y / q_{2}$ and $\tilde{\alpha}_{2}\left(z, \epsilon_{2}\right) \equiv$ $q_{2} \alpha_{2}\left(q_{2} z, \epsilon_{2}\right)$ we arrive at

$$
\frac{d \tilde{\alpha}_{2}\left(z, \epsilon_{2}\right)}{d z}+\tilde{\alpha}_{2}^{2}\left(z, \epsilon_{2}\right)=2\left(V_{1}(z)-\epsilon_{2}\right),
$$

where $V_{1}(z)$ is given by (25). A direct use of equations (10-12) with $i=2$ leads to a simple expression for the solution to Eq. (29) in terms of two solutions to (4) and (20) at the factorization energies $\epsilon_{1}$ and $\epsilon_{2}$, respectively

$$
\tilde{\alpha}_{2}\left(z, \epsilon_{2}\right)=-\tilde{\alpha}_{1}\left(z, \epsilon_{1}\right)-\frac{2\left(\epsilon_{1}-\epsilon_{2}\right)}{\tilde{\alpha}_{1}\left(z, \epsilon_{1}\right)-\tilde{\alpha}_{1}\left(z, \epsilon_{2}\right)} .
$$

According to (25), the potential $V_{2}(z)$ reads

$$
\begin{gathered}
V_{2}(z)=V_{1}(z)-\frac{d \tilde{\alpha}_{2}\left(z, \epsilon_{2}\right)}{d z} \\
=V_{0}(z)+\frac{d}{d z}\left[\frac{2\left(\epsilon_{1}-\epsilon_{2}\right)}{\tilde{\alpha}_{1}\left(z, \epsilon_{1}\right)-\tilde{\alpha}_{1}\left(z, \epsilon_{2}\right)}\right] .
\end{gathered}
$$

It has the same levels $E_{n}$ as $V_{0}(x)$ plus two additional ones at $\epsilon_{1}$ and $\epsilon_{2}$ ordered by simplicity as $\epsilon_{2}<\epsilon_{1}<E_{0}$. We notice that Samsonov has recently shown the possibility to construct physically relevant potentials (without singularities) by using a second order intertwining technique with $\epsilon_{1}>E_{0}$ and $\epsilon_{2}>E_{0}$ [29]. It is a simple matter now to construct a potential such that the corresponding Hamiltonian $\tilde{H}_{2}=\left(q_{1} q_{2}\right)^{-2} H_{2}$ acquires the standard Schrödinger form in the original coordinate $x$

$$
\begin{gathered}
\tilde{H}_{2}=-\frac{1}{2} \frac{d^{2}}{d x^{2}}+\tilde{V}_{2}(x), \\
\tilde{V}_{2}(x)=\frac{1}{\left(q_{1} q_{2}\right)^{2}} V_{2}\left(\frac{x}{q_{1} q_{2}}\right)=\frac{1}{\left(q_{1} q_{2}\right)^{2}} V_{0}\left(\frac{x}{q_{1} q_{2}}\right) \\
+\frac{1}{q_{1} q_{2}}\left[\frac{2\left(\epsilon_{1}-\epsilon_{2}\right)}{\tilde{\alpha}_{1}\left(\frac{x}{q_{1} q_{2}}, \epsilon_{1}\right)-\tilde{\alpha}_{1}\left(\frac{x}{q_{1} q_{2}}, \epsilon_{2}\right)}\right]^{\prime} .
\end{gathered}
$$

The generalized intertwining relationship involving $H_{0}$ and $\widetilde{H}_{2}$ is the following

$$
\tilde{H}_{2}\left(A_{\lambda_{2}}^{+} A_{\lambda_{1}}^{+}\right)=\left(q_{1} q_{2}\right)^{-2}\left(A_{\lambda_{2}}^{+} A_{\lambda_{1}}^{+}\right) H_{0} .
$$

Thus, the spectrum of $\tilde{H}_{2}$ is of the form $\left\{\left(q_{1} q_{2}\right)^{-2} \epsilon_{2},\left(q_{1} q_{2}\right)^{-2} \epsilon_{1},\left(q_{1} q_{2}\right)^{-2} E_{n}, n=0,1, \ldots\right\}$.

As we have been discussing along this paper, if we get solutions to the Riccati equations (4) and (20) for isolated values of $\epsilon_{1}$ and $\epsilon_{2}$, when varying the product $q_{1} q_{2}$ (by changing either $q_{1}$, or $q_{2}$, or both) in order to manipulate the spectra of $\tilde{V}_{2}(x)$ we will be just scaling the basic spectrum $\left\{\epsilon_{2}, \epsilon_{1}, E_{n}\right\}$ obtained from $H_{0}$ by performing two iterations of the standard intertwining with factorization energies $\epsilon_{1}$ and $\epsilon_{2}$. Once again, from the spectral engineering viewpoint the most interesting cases arise when we find solutions for $\epsilon_{1}$ and $\epsilon_{2}$ sweeping a real interval $I$. If this would be the case, by choosing for instance $\epsilon_{1}=q_{1}^{2} \epsilon_{1 \mathrm{~F}}$, where the three 
parameters $\epsilon_{1 \mathrm{~F}} \in I, q_{2}$ and $\epsilon_{2}$ are fixed, when varying $q_{1}$ so that always $\epsilon_{1} \in I$ and the ordering between $\epsilon_{1}$ and $\epsilon_{2}$ is maintained $\left(\epsilon_{2}<q_{1}^{2} \epsilon_{1 \mathrm{~F}}\right)$, the unscaled spectrum $\left\{\left(q_{2}^{-2} \epsilon_{2}\right),\left(q_{2}^{-2} \epsilon_{1 \mathrm{~F}}\right),\left(q_{2}^{-2} E_{n}\right)\right\}$ at $q_{1}=1$ will become scaled by the factor $q_{1}^{-2}$ except for the first excited level $\left(q_{2}^{-2} \epsilon_{1 \mathrm{~F}}\right)$ that will remain fixed. A similar static effect onto the ground state level can be obtained if we choose $\epsilon_{2}=q_{2}^{2} \epsilon_{2 \mathrm{~F}}$, where now the three fixed parameters are $\epsilon_{2 \mathrm{~F}}$, $q_{1}$ and $\epsilon_{1}$ : by changing $q_{2}$ so that $q_{2}^{2} \epsilon_{2 \mathrm{~F}}<\epsilon_{1}$ the excited levels at $q_{2}=1$ are scaled by the factor $q_{2}^{-2}$ while the ground state level remains fixed, as can be seen from the spectrum of $\tilde{V}_{2}(x),\left\{\left(q_{1}^{-2} \epsilon_{2 \mathrm{~F}}\right), q_{2}^{-2}\left(q_{1}^{-2} \epsilon_{1}\right), q_{2}^{-2}\left(q_{1}^{-2} E_{n}\right)\right\}$. A third interesting possibility arises by choosing $\epsilon_{1}=$ $\left(q_{1} q_{2}\right)^{2} \epsilon_{1 \mathrm{~F}}$ and $\epsilon_{2}=\left(q_{1} q_{2}\right)^{2} \epsilon_{2 \mathrm{~F}}$ with $\epsilon_{1 \mathrm{~F}}$ and $\epsilon_{2 \mathrm{~F}}$ fixed: when varying the product $q_{1} q_{2}$ by maintaining always $\epsilon_{1} \in I$ and $\epsilon_{2} \in I$, we will be basically scaling the spec$\operatorname{trum}\left\{\epsilon_{2 \mathrm{~F}}, \epsilon_{1 \mathrm{~F}}, E_{n}\right\}$ at $q_{1}=q_{2}=1$ by the factor $\left(q_{1} q_{2}\right)^{-2}$ except for the two fixed lowest levels $\epsilon_{2 \mathrm{~F}}$ and $\epsilon_{1 \mathrm{~F}}$, i.e., Spectrum $\left[\tilde{H}_{2}\right]=\left\{\epsilon_{2 \mathrm{~F}}, \epsilon_{1 \mathrm{~F}},\left(q_{1} q_{2}\right)^{-2} E_{n}\right\}$.

\section{EXAMPLE: THE HARMONIC OSCILLATOR}

Let us illustrate the spectral engineering effects previously discussed through the simple example of the harmonic oscillator potential $V_{0}(x)=x^{2} / 2$. The key point is to find the general solution to the Riccati equation (4) for different values of the factorization energy $\epsilon$. Let us notice that the case with $\epsilon=-1 / 2$ was solved for the first time by Mielnik [11]. For an arbitrary value of $\epsilon<1 / 2$, the general solution has been found by Sukumar [13], and a convenient expression in terms of confluent hypergeometric functions has been provided by Junker and Roy 22] (see also [18])

$$
\begin{aligned}
& \alpha(x, \epsilon)=x+\frac{d}{d x}\left\{\operatorname { l n } \left[{ }_{1} F_{1}\left(\frac{1+2 \epsilon}{4}, \frac{1}{2} ;-x^{2}\right)\right.\right. \\
& \left.\left.+2 \nu \frac{\Gamma\left(\frac{3-2 \epsilon}{4}\right)}{\Gamma\left(\frac{1-2 \epsilon}{4}\right)} x_{1} F_{1}\left(\frac{3+2 \epsilon}{4}, \frac{3}{2} ;-x^{2}\right)\right]\right\} .
\end{aligned}
$$

We have denoted the solution of (4) as $\alpha(x, \epsilon)$ instead of $\alpha_{1}(x, \epsilon)$ because this will simplify the discussion for all the intertwinings of this paper. We can distinguish four different possibilities of the spectral engineering.

(i). As it was discussed in section 2 , the potentials

$$
V_{1}(x)=\frac{x^{2}}{2}-\alpha_{1}^{\prime}\left(x, \epsilon_{1}\right),
$$

$\alpha_{1}\left(x, \epsilon_{1}\right) \equiv \alpha\left(x, \epsilon_{1}\right)$ as given by (35), have spectra $\left\{\epsilon_{1}, E_{n}=n+1 / 2, n=0,1, \cdots\right\}$. In order to avoid singularities in $V_{1}(x)$ we must have $\left|\nu_{1}\right|<1$, where $\nu_{1}$ denotes the $\nu$-constant of (35) associated to the factorization energy $\epsilon_{1}$. The simplest spectral manipulation consists in varying $\epsilon_{1}$ in the domain $(-\infty, 1 / 2)$ so that the ground state level of $V_{1}(x)$ is changing while the excited levels $E_{n}$ remain fixed [22]. This kind of spectral engineering is illustrated in figure 1 for $\nu_{1}=0.9$ : if the ground state energy of $H_{1}$ is moved, the potentials (36) have to change their form in order to maintain the excited levels $E_{n}$ at fixed positions. It is worth to notice that the potentials we are drawing are non-symmetric $\left(V_{1}(-x) \neq V_{1}(x)\right)$. The symmetric case arises for $\nu_{1}=0$ (see [22]).

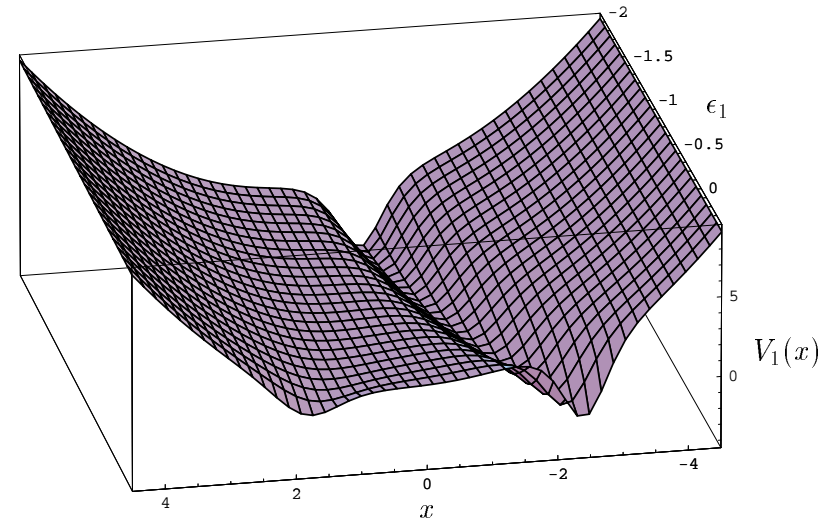

FIG. 1. Spectral manipulation of the potentials $V_{1}(x)$ of (36) as the ground state energy $\epsilon_{1}$ moves inside $\left[-2, \frac{1}{2}\right)$ and $\nu_{1}=0.9$. The excited levels $\left\{E_{n}=n+\frac{1}{2}, n=0,1, \ldots\right\}$ remain fixed for any $\epsilon_{1} \in\left(-\infty, \frac{1}{2}\right)$.

(ii). Consider now the iterations of the first order intertwining of section 3. In order to simplify the discussion, we restrict ourselves to two iterations. The potentials at the end of those two transformations turn into

$$
V_{2}(x)=\frac{x^{2}}{2}+\left[\frac{2\left(\epsilon_{1}-\epsilon_{2}\right)}{\alpha_{1}\left(x, \epsilon_{1}\right)-\alpha_{1}\left(x, \epsilon_{2}\right)}\right]^{\prime},
$$

where once again $\alpha_{1}\left(x, \epsilon_{1}\right) \equiv \alpha\left(x, \epsilon_{1}\right)$ and $\alpha_{1}\left(x, \epsilon_{2}\right) \equiv$ $\alpha\left(x, \epsilon_{2}\right)$. Notice that to each factorization energy $\epsilon_{i}$ an arbitrary constant $\nu_{i}, i=1,2$ is associated (see (35)). If the two new levels are ordered as $\epsilon_{2}<\epsilon_{1}<1 / 2$, the domains of $\nu_{1}$ and $\nu_{2}$ have to be restricted to $\left|\nu_{1}\right|<1$ and $\left|\nu_{2}\right|>1$ in order to avoid singularities in $V_{2}(x)$.

The spectral engineering can be implemented by varying $\epsilon_{2}, \epsilon_{1}$, or both. If we sweep $\epsilon_{2}$ and maintain fixed $\epsilon_{1}$, we obtain again the kind of manipulation described at issue (i) consisting in fixing the excited levels of $V_{2}(x)$ and moving only the ground state energy. A physically different situation arises if $\epsilon_{2}$ is static but $\epsilon_{1}$ changes, i.e., the first excited level of $V_{2}(x)$ is moving. This kind of spectral manipulation is illustrated in figure 2 for $\epsilon_{1} \in[-0.4,0.4], \epsilon_{2}=-1 / 2, \nu_{1}=0$ and $\nu_{2}=10000$. The fixed levels are placed at $\{-1 / 2,1 / 2,3 / 2, \ldots\}$. Notice the 
almost symmetric nature of $V_{2}(x)\left(V_{2}(-x) \approx V_{2}(x)\right)$; the strict symmetry is achieved for $\nu_{1}=0$ and $\left|\nu_{2}\right| \rightarrow \infty$. We also notice the triple well feature, characteristic of some of the potentials derived by the iteration of two first order intertwinings. For higher orders of iteration it is possible to design more complicated multiple well potentials. As far as we know, this is the first time that potentials where the first excited level is movable have been derived. Moreover, a more general manipulation procedure can be designed by changing $\epsilon_{1}$ and $\epsilon_{2}$ independently to each other. In such a case, the fixed part of the spectrum $\{3 / 2,5 / 2, \ldots\}$ will start from the second excited state while the first two energy levels will be moving according to the choice of the spectral manipulator.

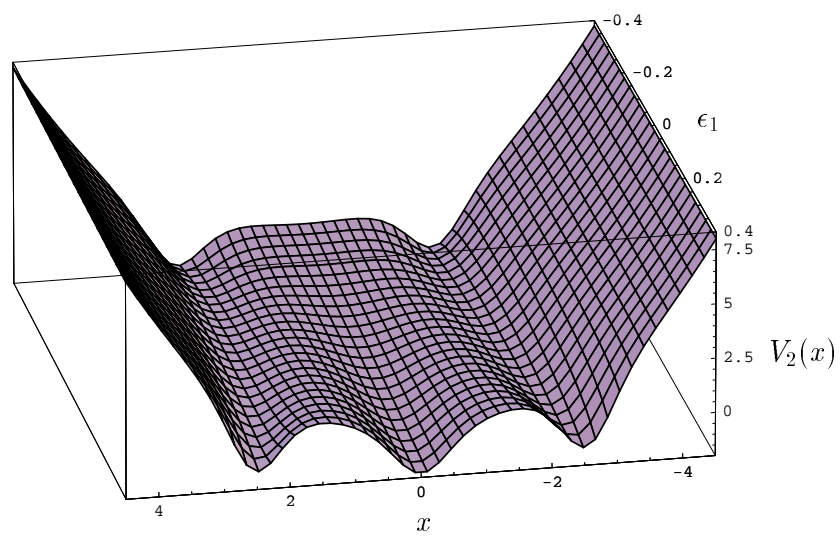

FIG. 2. Spectral manipulation of the potentials $V_{2}(x)$ of (37) as the first excited level $\epsilon_{1}$ moves inside $[-0.4,0.4]$ and $\nu_{1}=0, \nu_{2}=10000$. The fixed part of the spectrum consists of the levels $\left\{-\frac{1}{2}, \frac{1}{2}, \frac{3}{2}, \ldots\right\}$.

(iii). Let us analyze now the spectral engineering possibilities offered by the scaling intertwining of section 4 . The potentials generated by means of this technique from the oscillator potential read:

$$
\tilde{V}_{1}(x)=q_{1}^{-4} \frac{x^{2}}{2}-q_{1}^{-1} \tilde{\alpha}_{1}^{\prime}\left(x / q_{1}, \epsilon_{1}\right),
$$

where $\tilde{\alpha}_{1}\left(x, \epsilon_{1}\right) \equiv \alpha\left(x, \epsilon_{1}\right)$. The spectrum of $\tilde{V}_{1}(x)$ is of the form $\left\{q_{1}^{-2} \epsilon_{1}, q_{1}^{-2}(n+1 / 2), n=0,1, \ldots\right\}$. By taking $\epsilon_{1}=q_{1}^{2} \epsilon_{1 \mathrm{~F}} \in I_{1}=(-\infty, 0), \epsilon_{1 \mathrm{~F}} \in I_{1}$ is fixed, and varying the scaling parameter $q_{1}$, the spectrum of $\tilde{V}_{1}(x)$ will be changing as $\left\{\epsilon_{1 \mathrm{~F}}, q_{1}^{-2}(n+1 / 2), n=0,1, \ldots\right\}$, i.e., the (excited) levels $E_{n}=n+1 / 2$ at $q_{1}=1$ will be scaled by the factor $q_{1}^{-2}$, but the ground state level will remain fixed. A similar treatment can be implemented for $\epsilon_{1} \in I_{2}=(0,1 / 2)$. A representation of this kind of spectral manipulation on $\tilde{V}_{1}(x)$ as $q_{1}$ is changed, with $\epsilon_{1 \mathrm{~F}}=-1 / 2$ and $\nu_{1}=0$, is shown in figure 3 . Notice that this choice of $\epsilon_{1 \mathrm{~F}}$ and $\nu_{1}$ ensures that, up to a displacement of the energy origin, the oscillator potential arises for $q_{1}=1$, and also that $\tilde{V}_{1}(x)$ is symmetric with respect to $x=0$. For $q_{1} \in(0,1)$ the potentials $\tilde{V}_{1}(x)$ have a double well, and the spacing between the excited levels $E_{n}=n+1 / 2$ at $q_{1}=1$ is expanded by the factor $q_{1}^{-2}$ while the ground state level is fixed at $\epsilon_{1 \mathrm{~F}}=-1 / 2$. On the other hand, for $q_{1} \in(1, \infty)$, the potentials $\tilde{V}_{1}(x)$ present a single well centered at $x=0$, which can be interpreted as a deformation of the oscillator bottom in order to maintain fixed the ground state energy level at $\epsilon_{1 \mathrm{~F}}=-1 / 2$. The excited levels $E_{n}=n+1 / 2$ at $q_{1}=1$ are 'squeezed' by the factor $q_{1}^{-2}$.

It is important to remark once again the opposite physical mechanisms of the spectral engineering taking place for the scaling intertwining (the ground state is fixed and the excited levels are changing) and for the standard intertwining technique (the ground state is changing and the excited levels are fixed).

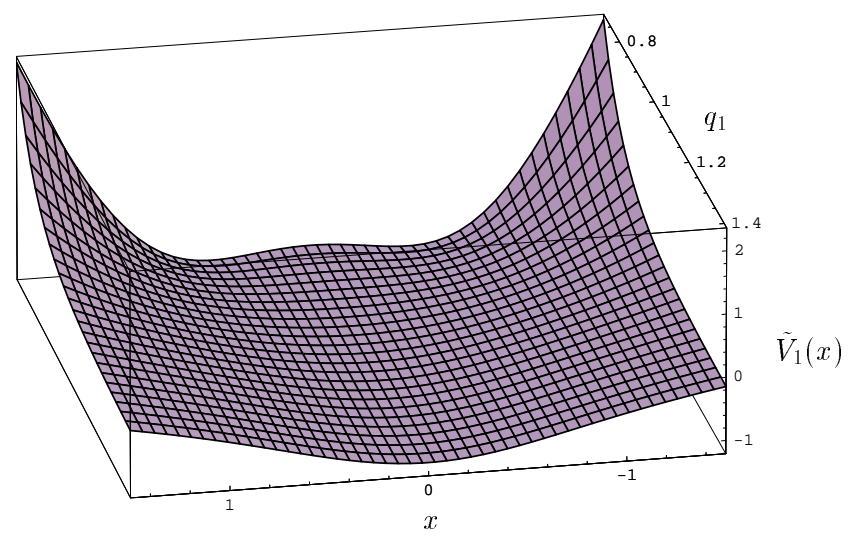

FIG. 3. Spectral engineering of the potentials $\tilde{V}_{1}(x)$ of (38) as $q_{1}$ moves inside $[1 / \sqrt{2}, \sqrt{2}]$ with $\nu_{1}=0$ and $\epsilon_{1}=-q_{1}^{2} / 2$.

The ground state energy is fixed at $\epsilon_{1 \mathrm{~F}}=-\frac{1}{2}$, and the

unscaled excited levels $E_{n}=n+\frac{1}{2}, n=0,1, \ldots$ are 'expanded' or 'squeezed' by the factor $q_{1}^{-2}$ for $0<q_{1}<1$ or $q_{1}>1$, respectively.

(iv). Let us discuss briefly the spectral manipulations achievable by the iterative scaling intertwining of section 5 . The potentials $\tilde{V}_{2}(x)$ after two iterations of the scaling intertwining with $V_{0}(x)=x^{2} / 2$ take the form

$$
\begin{aligned}
& \tilde{V}_{2}(x)=\frac{1}{\left(q_{1} q_{2}\right)^{2}} V_{2}\left(\frac{x}{q_{1} q_{2}}\right)=\left(q_{1} q_{2}\right)^{-4} \frac{x^{2}}{2} \\
& +\frac{1}{q_{1} q_{2}}\left[\frac{2\left(\epsilon_{1}-\epsilon_{2}\right)}{\tilde{\alpha}_{1}\left(\frac{x}{q_{1} q_{2}}, \epsilon_{1}\right)-\tilde{\alpha}_{1}\left(\frac{x}{q_{1} q_{2}}, \epsilon_{2}\right)}\right]^{\prime},
\end{aligned}
$$


where $\tilde{\alpha}_{1}\left(x, \epsilon_{1}\right) \equiv \alpha\left(x, \epsilon_{1}\right), \quad \tilde{\alpha}_{1}\left(x, \epsilon_{2}\right) \equiv \alpha\left(x, \epsilon_{2}\right)$, $\epsilon_{2}<\epsilon_{1}<1 / 2,\left|\nu_{1}\right|<1$ and $\left|\nu_{2}\right|>1$. The spectrum of those potentials is of the form $\left\{\left(q_{1} q_{2}\right)^{-2} \epsilon_{2},\left(q_{1} q_{2}\right)^{-2} \epsilon_{1},\left(q_{1} q_{2}\right)^{-2}(n+1 / 2), n=0,1, \ldots\right\}$. Let us notice that the potentials (39) form a sixparameter family labeled by $\epsilon_{1}, q_{1}, \nu_{1}, \epsilon_{2}, q_{2}, \nu_{2}$, although the spectra does dot depend on the values of $\nu_{1}$ and $\nu_{2}$ except for the fact that they have to be taken in the domain for which $\tilde{V}_{2}(x)$ has no singularities. The presence of so many parameters provides us with various possibilities for manipulating quantum spectra, as discussed at the end of section 5. For instance, if we take $\epsilon_{1}=q_{1}^{2} \epsilon_{1 \mathrm{~F}}$, with $\epsilon_{1 \mathrm{~F}}, q_{2}$ and $\epsilon_{2}$ fixed, by varying $q_{1}$ we will be manufacturing potentials with spectra of the type $\left\{q_{2}^{-2} \epsilon_{2}, q_{2}^{-2} \epsilon_{1 \mathrm{~F}}, q_{2}^{-2}(n+1 / 2), n=0,1, \ldots\right\}$ scaled by the factor $q_{1}^{-2}$ excepting the first excited level $q_{2}^{-2} \epsilon_{1 \mathrm{~F}}$, which is maintained fixed (see the illustration of this effect on figure 4). Notice that this behaviour is opposite to the one illustrated in figure 2 where the first excited level is changing and the remaining part of the spectrum is fixed.

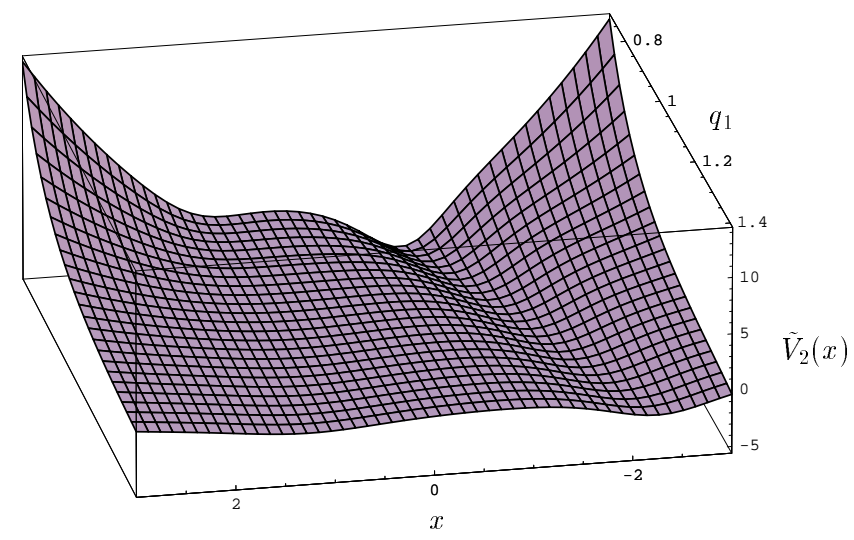

FIG. 4. Spectral manipulation of $\tilde{V}_{2}(x)$ as $q_{1}$ moves inside $[1 / \sqrt{2}, \sqrt{2}], \nu_{1}=0, \nu_{2}=1.1, \epsilon_{1}=-\frac{q_{1}^{2}}{2}, \epsilon_{2}=-\frac{3}{2}$ and $q_{2}=1$. The first excited level is fixed at $\epsilon_{1 \mathrm{~F}}=-\frac{1}{2}$, while the other levels $\left\{-\frac{3}{2}, \frac{1}{2}, \frac{5}{2}, \ldots\right\}$ are scaled by the factor $q_{1}^{-2}$.

A different possibility arises by taking $\epsilon_{1}=$ $\left(q_{1} q_{2}\right)^{2} \epsilon_{1 \mathrm{~F}}, \epsilon_{2}=\left(q_{1} q_{2}\right)^{2} \epsilon_{2 \mathrm{~F}}$; when varying the product $q_{1} q_{2}$ we will be scaling the spectrum at $q_{1}=q_{2}=1$, $\left\{\epsilon_{1 \mathrm{~F}}, \epsilon_{2 \mathrm{~F}}, E_{n}=n+1 / 2\right\}$, by the factor $\left(q_{1} q_{2}\right)^{-2}$ excepting the lowest two levels $\epsilon_{1 \mathrm{~F}}$ and $\epsilon_{2 \mathrm{~F}}$ that remain static. An illustration of this effect is presented in figure 5 for $\epsilon_{1 \mathrm{~F}}=\frac{1}{4}, \epsilon_{2 \mathrm{~F}}=-\frac{1}{2}, \nu_{1}=0, \nu_{2}=10000, q_{2}=1$ and $q_{1} \in\left[\frac{1}{\sqrt{2}}, \sqrt{2}\right)$.

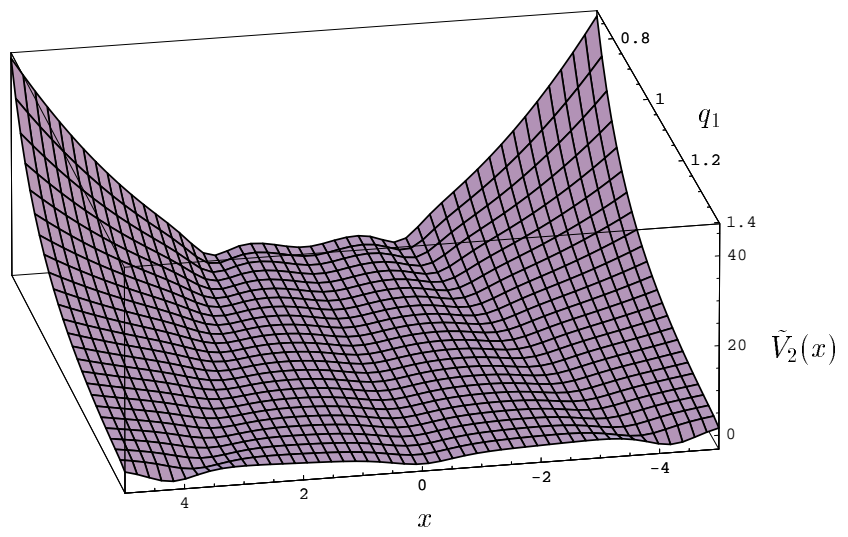

FIG. 5. Spectral engineering of $\tilde{V}_{2}(x)$ for $q_{1}$ in $[1 / \sqrt{2}, \sqrt{2})$, $\nu_{1}=0, \nu_{2}=10000, \epsilon_{1}=\frac{q_{1}^{2}}{4}, \epsilon_{2}=-\frac{q_{1}^{2}}{2}$ and $q_{2}=1$. The two lowest levels are fixed at $\epsilon_{1 \mathrm{~F}}=\frac{1}{4}$ and $\epsilon_{2 \mathrm{~F}}=-\frac{1}{2}$, while the other ones $\left\{\frac{1}{2}, \frac{3}{2}, \ldots\right\}$ are scaled by the factor $q_{1}^{-2}$.

\section{CONCLUDING REMARKS}

We presented some of the spectral engineering possibilities offered by the scaling intertwining procedure. Since this method is based on more parameters with respect to the standard ones it can describe a wealth of technological and experimental situations. For this, it is sufficient to have a clear physical meaning of the parameters. For example, the scaling parameter can be associated to self-similar (fractal) properties of micro and nanocavities, whereas the $\nu$ parameters are a measure of finite-size and boundary effects on the quantum spectra [30].

Before closing, we mention that in interesting works, Spiridonov 25] has used a rather similar type of scaling intertwining for emphasizing the connection between the $q$-deformed calculus and Shabat's infinite chain of reflectionless potentials [24]. In such a treatment the so-called self-similar potentials have been introduced and characterized, and at the same time the corresponding spectrum has been determined by means of the algebraic technique itself. On the other hand, in this paper the orientation has been different, namely to take initially a potential with known spectrum in order to generate a new potential with known and different (in general) spectrum. When we make use of the freedom provided by the different parameters of the families of potentials generated by means of the various intertwinings (standard and/or scaled ones), we arrive at the spectral engineering process widely discussed in this paper. 


\section{ACKNOWLEDGEMENTS}

This work was supported in part by the CONACyT Projects 32086-E and 458100-5-25844E. HCR wishes to thank for the kind hospitality at the Departamento de Física, CINVESTAV.

[1] M.A. Kastner, Rev. Mod. Phys. 64, 849 (1992); L.P. Kouwenhoven, N.C. van der Vaart, A.T. Johnson, W. Kool, C.J.P.M. Harmans, J.G. Williamson, A.A.M. Staring and C.T. Foxon, Z. Phys. B 85, 367 (1991); A.P. Heberle, W.W. Rühle and K. Ploog, Phys. Rev. Lett. 72, 3887 (1994).

[2] S. Chu, Rev. Mod. Phys. 70, 685 (1998); C. CohenTannoudji, Rev. Mod. Phys. 70, 707 (1998); W.D. Phillips, Rev. Mod. Phys. 70, 721 (1998).

[3] B. Mielnik and D.J. Fernández, J. Math. Phys. 30, 537 (1989).

[4] C. Zicovich-Wilson, W. Jaskólski and J.H. Planelles, Int. J. Quantum Chem. 54, 661 (1995).

[5] S. Haroche, in AIP Conference Proceedings 464, S. Hacyan et. al. Eds, American Institute of Physics, New York (1999), p. 45.

[6] D.J. Fernández, Nuovo Cim. B 107, 885 (1992); D.J. Fernández and B. Mielnik, J. Math. Phys. 35, 2083 (1994).

[7] P. Gross, V. Ramakrishna, E. Vilallonga, H. Rabitz, M. Littman, S.A. Lyon and M. Shayegan, Phys. Rev. B 49, 11100 (1994); V. Ramakrishna, M.V. Salapaka, M. Dahleh, H. Rabitz and A. Peirce, Phys. Rev. A 51, 960 (1995).

[8] P. Brumer and M. Shapiro, Annu. Rev. Phys. Chem. 43, 257 (1992); A. Haché, Y. Kostoulas, R. Atanasov, J.L.P. Hughes, J.E. Sipe and H.M. van Driel, Phys. Rev. Lett. 78, 306 (1997); M.U. Wehner, M.H. Ulm, D.S. Chemla and M. Wegner, Phys. Rev. Lett. 80, 1992 (1998).

[9] V.M. Chabanov and B.N. Zakhariev, Phys. Rev. A 49, 3159 (1994); B.N. Zakhariev and V.M. Chabanov, Inv. Prob. 13, R47 (1997).

[10] L. Infeld and T.E. Hull, Rev. Mod. Phys. 23, 21 (1951).

[11] B. Mielnik, J. Math. Phys. 25, 3387 (1984).

[12] D.J. Fernández, Lett. Math. Phys. 8, 337 (1984); D.J. Fernández, J. Negro and M.A. del Olmo, Ann. Phys. 252, 386 (1996).

[13] E. Witten, Nucl. Phys. B 185, 513 (1981); M.M. Nieto, Phys. Lett. B 145, 208 (1984); C.V. Sukumar, J. Phys. A 18, 2917 (1985).

[14] F. Cooper, A. Khare and U. Sukhatme, Phys. Rep. 251, 267 (1995).

[15] H.C. Rosu and M.A. Reyes, Phys. Rev. E 51, 5112 (1995); H.C. Rosu, Phys. Rev. A 54, 2571 (1996); H.C. Rosu, Phys. Rev. E 56, 2269 (1997); H.C. Rosu and M.A. Reyes, Phys. Rev. E 57, 4850 (1998).

[16] A.A. Andrianov, M.V. Ioffe and V. Spiridonov, Phys. Lett. A 174, 273 (1993); A.A. Andrianov, M.V. Ioffe,
F. Cannata and J.P. Dedonder, Int. J. Mod. Phys. A 10, 2683 (1995).

[17] V.G. Bagrov and B.F. Samsonov, Theor. Math. Phys. 104, 1051 (1995); V.G. Bagrov and B.F. Samsonov, Phys. Part. Nucl. 28, 374 (1997).

[18] D.J. Fernández, Int. J. Mod. Phys. A 12, 171 (1997); D.J. Fernández, M.L. Glasser and L.M. Nieto, Phys. Lett. A 240, 15 (1998); D.J. Fernández, V. Hussin and B. Mielnik, Phys. Lett. A 244, 309 (1998); D.J. Fernández and V. Hussin, J. Phys. A 32, 3603 (1999).

[19] J.O. Rosas-Ortiz, J. Phys. A 31, L507 (1998); J.O. Rosas-Ortiz, J. Phys. A 31, 10163 (1998).

[20] B. Mielnik, L.M. Nieto and O. Rosas-Ortiz, Phys. Lett. A 269, 70 (2000).

[21] I.F. Márquez, J. Negro and L.M. Nieto, J. Phys. A 31, 4115 (1998).

[22] G. Junker and P. Roy, Phys. At. Nucl. 61, 1736 (1998); G. Junker and P. Roy, Ann. Phys. 270, 155 (1998).

[23] P.A. Deift, Duke Math. J. 45, 267 (1978); A. Anderson, Phys. Rev. A 43, 4602 (1991).

[24] A. Shabat, Inv. Prob. 8, 303 (1992).

[25] V. Spiridonov, Phys. Rev. Lett. 69, 398 (1992); V. Spiridonov, Mod. Phys. Lett. A 7, 1241 (1992).

[26] V. Spiridonov, Phys. Rev. A 52, 1909 (1995).

[27] V.E. Adler, Funct. Anal. Appl. 27, 141 (1993).

[28] J. Negro, L.M. Nieto and O. Rosas-Ortiz, J. Phys. A 33, 7207 (2000).

[29] B.F. Samsonov, Phys. Lett. A 263, 274 (1999).

[30] C. Monthus, G. Oshanin, A. Comtet and S. Burlatsky, Phys. Rev. E 54, 231 (1996); L.J. Boya, H.C. Rosu, A.J. Seguí-Santonja, J. Socorro and F.J. Vila, J. Phys. A 31, 8835 (1998). 